ANNALES

POLONICI MATHEMATICI

XII (1962)

\title{
On a generalization of Simpson's formula
}

\author{
by R. B. Saxena (Lucknow, India)
}

1. Recently S. Goląb has proposed and solved certain interesting problems related to the well-known Simpson's formula. The object of this paper is to give a generalization of Simpson's formula by taking as our starting point a mean-value theorem of Cioranescu. We later propose some problems in the light of Golab's results and resolve them in the general case providing thereby at the same time a generalization of Goląb's results.

We know the following formula, due to Cioranescu:

$$
\int_{a}^{b} f(x) P_{n}(x) d x=\frac{f^{(n)}(\xi)}{(n+1) !} \int_{a}^{b} \ldots \int_{a}^{b} V_{n+1}^{2} d v_{n+1} \quad(a<\xi<b),
$$

where $P_{n}(x)$ is a Legendre polynomial over the interval $(a, b), d v_{n+1}$ $=d x_{0} d x_{1} \ldots d x_{n}$ and $V_{n+1}$ is the Vandermonde's determinant $V_{n+1}\left(x_{0} x_{1} \ldots x_{n}\right)$ $=\operatorname{Det}\left(x_{k} \ldots x_{k}^{n}\right)$. The problem naturally arises whether we can give an analogue of Simpson's formula for the above integral on the left.

We may take the interval $(0, h)$ instead of $(a, b)$ without loss of generality, and let us write

$$
\int_{0}^{h} f(x) P_{n}(x) d x=h^{n+1}\left\{\lambda_{0} f^{(n)}(0)+\lambda_{1} f^{(n)}\left(\frac{h}{2}\right)+\lambda_{2} f^{(n)}(h)\right\},
$$

a formula easily suggested by (1). Let

$$
f(x)=\sum_{i=0}^{\infty} a_{i} x^{d} .
$$

Since over the interval $(0, h), P_{n}(x)$ is given by

$$
P_{n}(x)=\left(\frac{h}{2}\right)^{n} \frac{1}{2^{n} n !} \cdot \frac{d^{n}}{d x^{n}}\left\{\frac{4}{h^{2}}\left(x-\frac{h}{2}\right)^{2}-1\right\}^{n} .
$$


We can easily find the following result:

$$
\int_{0}^{n} \infty^{n+p} P_{n}(x) d x=\frac{\{\Gamma(n+p+1)\}^{2}}{\Gamma(p+1) \Gamma(2 n+p+2)} h^{n+p+1} .
$$

From this one can easily find the coefficients $\lambda_{0}, \lambda_{1}, \lambda_{2}$ in (2) and we have

$$
\lambda_{0}=\lambda_{2}=\frac{\Gamma(n+2)}{\Gamma(2 n+4)} \quad \text { and } \quad \lambda_{1}=4(n+1) \frac{\Gamma(n+2)}{\Gamma(2 n+4)}
$$

We thus establish the formula

$$
\begin{aligned}
& \int_{0}^{h} f(x) P_{n}(x) d x \\
& =\frac{\Gamma(n+2)}{\Gamma(2 n+4)} h^{n+1}\left[f^{(n)}(0)+4(n+1) f^{(n)}\left(\frac{h}{2}\right)+f^{(n)}(h)\right]+R(h),
\end{aligned}
$$

where

$$
R(h)=-\frac{n+1}{48} \cdot \frac{\Gamma(n+3)}{\Gamma(2 n+6)} h^{n+s} f^{(n+6)}(\xi) \quad(0<\xi<h) .
$$

For $n=0,(4)$ and (5) give Simpson's formula and the remainder thereof.

2. Putting $T(h)=\int_{0}^{h} f(x) P_{n}(x) d x$ and

$$
T_{1}\left(h ; \lambda_{0}, \lambda_{1}, \lambda_{2}\right)=h^{n+1}\left[\lambda_{0} f^{(n)}(0)+\lambda_{1} f^{(n)}(\theta h)+\lambda_{2} f^{(n)}(h)\right] \quad(0<\theta<1)
$$

we now propose to determine $\lambda_{0}, \lambda_{1}, \lambda_{2}$ such that $R(h) \equiv T(h)-T_{1}(h$; $\left.\lambda_{0}, \lambda_{1}, \lambda_{2}\right)$ is of the greatest possible order of smallness. Let $f(x)$ be regular in the neighbourhood of the origin having the expansion

$$
\text { (A) } \begin{aligned}
f(h)=f(0)+a_{1} h+\ldots+a_{n} h^{n} & +a_{n+p} h^{n+p}+ \\
& +a_{n+q} h^{n+q}+a_{n+p} h^{n+r}+a_{n+s} h^{n+s}+\ldots
\end{aligned}
$$

where $a_{n+p}, a_{n+q}, a_{n+r}, a_{n+s} \neq 0$ and $1 \leqslant p<q<r<8 \ldots$ We easily get the following equations to determine $\lambda_{0}, \lambda_{1}, \lambda_{2}$ :

$$
\begin{aligned}
& \lambda_{0}+\lambda_{1}+\lambda_{2}=\frac{\Gamma(n+1)}{\Gamma(2 n+2)}, \\
& \theta^{p} \lambda_{1}+\lambda_{2}=\frac{\Gamma(p+n+1)}{\Gamma(p+2 n+2)}, \\
& \theta^{q} \lambda_{1}+\lambda_{2}=\frac{\Gamma(q+n+1)}{\Gamma(q+2 n+2)} .
\end{aligned}
$$


Equations (7) and (8) determine $\lambda_{1}, \lambda_{2}$ uniquely as

$$
W=\left|\begin{array}{ll}
\theta^{p} & 1 \\
\theta^{q} & 1
\end{array}\right|=\theta^{p}-\theta^{q}>0 \quad(p<q)
$$

and $\lambda_{0}$ is then determined by (6). The remainder is then of the order of smallest of $h^{r+1+n}$.

3. The next question naturally is: Can we choose $\theta$ in such a way that the order of smallness of $R(h)$ is further increased? In that case we will require, along with (6), (7), and (8), the following relation:

$$
\theta^{r} \lambda_{1}+\lambda_{2}=\frac{\Gamma(r+n+1)}{\Gamma(r+2 n+2)} .
$$

The condition of consistency of (7), (8), and (9) gives

$$
\Delta(\theta) \equiv\left|\begin{array}{ccc}
\theta^{p} & 1 & \frac{\Gamma(p+n+1)}{\Gamma(p+2 n+2)} \\
\theta^{q} & 1 & \frac{\Gamma(q+n+1)}{\Gamma(q+2 n+2)} \\
\theta^{r} & 1 & \frac{\Gamma(r+n+1)}{\Gamma(r+2 n+2)}
\end{array}\right|=0 .
$$

Putting $\alpha=q-p, \beta=r-q,(10)$ can be written thus:

$$
\begin{aligned}
\Delta(\theta) \equiv\left\{\frac{\Gamma(p+n+1)}{\Gamma(p+2 n+2)}-\frac{\Gamma(q+n+1)}{\Gamma(q+2 n+2)}\right\} \theta^{\alpha+\beta}- \\
\quad-\left\{\frac{\Gamma(p+n+1)}{\Gamma(p+2 n+2)}-\frac{\Gamma(r+n+1)}{\Gamma(r+2 n+2)}\right\} \theta^{\alpha}+ \\
+\left\{\frac{\Gamma(q+n+1)}{\Gamma(q+2 n+2)}-\frac{\Gamma(r+n+1)}{\Gamma(r+2 n+2)}\right\}=0 .
\end{aligned}
$$

Equation (11) is an algebraic equation in $\theta$ of degree $a+\beta$. We shall show that this equation has a unique root $\theta$ in the open interval $(0,1)$. Since

$$
\begin{array}{r}
\Delta^{\prime}(\theta)=\theta^{\alpha-1}\left[(\alpha+\beta)\left\{\frac{\Gamma(p+n+1)}{\Gamma(p+2 n+2)}-\frac{\Gamma(q+n+1)}{\Gamma(q+2 n+2)}\right\} \theta^{\theta}-\right. \\
\left.-\alpha\left\{\frac{\Gamma(p+n+1)}{\Gamma(p+2 n+2)}-\frac{\Gamma(r+n+1)}{\Gamma(r+2 n+2)}\right\}\right],
\end{array}
$$

we have

$$
\begin{gathered}
\Delta(0)>0, \quad \Delta(1)=0 \quad \text { and } \\
\Delta^{\prime}(1)=\frac{\beta}{(p+n+1)_{n+1}}-\frac{a+\beta}{(q+n+1)_{n+1}}+\frac{\alpha}{(r+n+1)_{n+1}}
\end{gathered}
$$

where $(p+n+1)_{n+1}=(p+n+1)(p+n+2) \ldots(p+2 n+1)$. 
Now in order to prove that $\Delta^{\prime}(1)>0$, we observe that

$$
\begin{aligned}
\Lambda^{\prime}(1)= & \frac{1}{(r+n+1)_{n+1}}\left\{\beta\left(1+\frac{a+\beta}{p+n+1}\right) \ldots\left(1+\frac{a+\beta}{p+2 n+1}\right)+\right. \\
& \left.+a-(a+\beta)\left(1+\frac{\beta}{q+n+1}\right) \ldots\left(1+\frac{\beta}{q+2 n+1}\right)\right\} \\
= & \frac{1}{(r+n+1)_{n+1}}\left[\beta(a+\beta)\left\{S_{1}+(\alpha+\beta) S_{2}+\ldots\right\}-\right. \\
& \left.-(\alpha+\beta) \beta\left\{s_{1}+\beta s_{2}+\ldots\right\}\right]
\end{aligned}
$$

where $S_{\nu}$ is the sum of the products, taken $v$ at a time, of $\frac{1}{p+n+1}, \ldots$, $\frac{1}{p+2 n+1}$ and $s_{v}$ is the sum $S_{v}$ where $p$ is replaced by $q$. Obviously $S,>s_{v}$ since $q>p$, and hence we have $\Delta^{\prime}(1)>0$.

From this and from (12) it follows that $\Delta(\theta)<0$ in a small neighbourhood of $\theta=1$ on the left. Also the unique root of $\Delta^{\prime}(\theta)=0$ is given by

$$
\theta^{\beta}=\frac{a}{a+\beta} \cdot \frac{(r+n+1)_{n+1}-(p+n+1)_{n+1}}{(q+n+1)_{n+1}-(p+n+1)_{n+1}} \cdot \frac{(q+n+1)_{n+1}}{(r+n+1)_{n+1}} .
$$

In order to show that the expression on the right is necessarily less than unity, we write it as follows:

(*) $\quad a\left\{\frac{1}{(p+n+1)_{n+1}}-\frac{1}{(r+n+1)_{n+1}}\right\}$

Now

$$
\div(\alpha+\beta)\left\{\frac{1}{(p+n+1)_{n+1}}-\frac{1}{(q+n+1)_{n+1}}\right\} \text {. }
$$

$$
\frac{1}{(p+k)_{n+1}}=\frac{1}{n !} \sum_{i=1}^{n}(-1)^{i}\left(\begin{array}{l}
n \\
i
\end{array}\right) \frac{1}{p+k+i}
$$

so that

$$
\begin{aligned}
& (a+\beta)\left\{\frac{1}{(p+n+1)_{n+1}}-\frac{1}{(q+n+1)_{n+1}}\right\} \\
& =\frac{a(a+\beta)}{n !} \sum_{i=0}^{n}(-1)^{i}\left(\begin{array}{c}
n \\
i
\end{array}\right) \frac{1}{(q+n+i+1)(p+n+i+1)} \\
& =\frac{a(a+\beta)}{n !} \int_{0}^{1} \int_{0}^{1} x^{p+n} y^{a+n}(1-x y)^{n} d x d y .
\end{aligned}
$$

Hence (13) reduces to

$$
\theta^{\beta}=\frac{I_{p r}^{n}}{I_{p q}^{n}}
$$


where

$$
I_{p q}^{n}=\int_{0}^{1} \int_{0}^{1} x^{p+n} y^{q+n}(1-x y)^{n} d x d y
$$

Obviously $I_{p q}^{n}>I_{p r}^{n}$ where $r>q$, since

$$
I_{p q}^{n}-I_{p r}^{n}=\int_{0}^{1} \int_{0}^{1} x^{p+n} y^{n}\left(y^{q}-y^{r}\right)(1-x y)^{n} d x d y>0 .
$$

We have thus proved that there is a choice of $\theta$ for which $R(h)$ is infinitely small of order $n+r+2$ at least. From the form $(\mathrm{A})$ of $f(h)$ it follows that $R(h)$ will be infinitely small of order $n+s+1$ at least.

4. Let the integral

$$
T(h)=\int_{0}^{h} f(x) P_{n}(x) d x
$$

of Art. 2 be approximated by

$$
T_{2}(h)=h^{n+1}\left\{\lambda_{0} f^{(n)}(0)+\lambda_{1} f^{(n)}\left(\theta_{1} h\right)+\lambda_{2} f^{(n)}\left(\theta_{2} h\right)\right\}
$$

where $0<\theta_{1} \leqslant \frac{1}{2}<\theta_{2} \leqslant 1$. For $\theta_{1}=\frac{1}{2}, \theta_{2}=1$, we get the same formula (2) of Art. 1, which for $n=0$ is Simpson's formula.

We then propose the following problem:

Determine the values of $\lambda_{0}, \lambda_{1}, \lambda_{2}$ so that for given $\theta_{1}$ and $\theta_{2}\left(0<\theta_{1}\right.$ $\leqslant \frac{1}{2}<\theta_{2} \leqslant 1$ ) the remainder is of the greatest order of smallness with respect to $h$.

Let $f(x)$ be regular in the neighbourhood of the origin having the development (A) of Art. 2. We easily get the following equations to determine $\lambda_{0}, \lambda_{1}, \lambda_{2}$ :

$$
\begin{gathered}
\lambda_{0}+\lambda_{1}+\lambda_{2}=\frac{\Gamma(n+1)}{\Gamma(2 n+2)}, \\
\lambda_{1} \theta_{1}^{p}+\lambda_{2} \theta_{2}^{p}=\frac{\Gamma(p+n+1)}{\Gamma(p+2 n+2)}, \\
\lambda_{1} \theta_{1}^{q}+\lambda_{2} \theta_{2}^{g}=\frac{\Gamma(q+n+1)}{\Gamma(q+2 n+2)} .
\end{gathered}
$$

Equations (16) and (17) determine $\lambda_{1}, \lambda_{2}$ uniquely, as

$$
W=\left|\begin{array}{cc}
\theta_{1}^{p} & \theta_{2}^{p} \\
\theta_{1}^{q} & \theta_{2}^{q}
\end{array}\right|=\theta_{1}^{p} \theta_{2}^{q}-\theta_{1}^{q} \theta_{2}^{p}=\theta_{1}^{p} \theta_{2}^{p}\left(\theta_{2}^{q-p}-\theta_{1}^{q-p}\right) \neq 0 \quad\left(0<\theta_{1}<\theta_{2} \leqslant 1\right) .
$$

We have thus proved that: 
If $\theta_{1}$ and $\theta_{2}$ are given numbers $\left(0<\theta_{1}<\theta_{2}<1\right)$ and $\lambda_{1}, \lambda_{2}$ are given by (16) and (17) and $\lambda_{0}$ by (15), then $R(h)$ is of the order of smallness $r+n+1$.

5. Naturally the following problem now arises:

Can we further raise the order of smallness of $R(h)$ by a proper choice of $\theta_{2}$ ?

Then, along with (15), (16) and (17), the following relation must be satisfied:

$$
\lambda_{1} \theta_{1}^{r}+\lambda_{2} \theta_{2}^{r}=\frac{\Gamma(r+n+1)}{\Gamma(r+2 n+2)} .
$$

The condition of consistency of (16), (17) and (18) then requires that

$$
\Delta(\theta) \equiv\left|\begin{array}{ccc}
\theta_{1}^{p} & \theta^{p} & \frac{\Gamma(p+n+1)}{\Gamma(p+2 n+2)} \\
\theta_{1}^{q} & \theta^{q} & \frac{\Gamma(q+n+1)}{\Gamma(q+2 n+2)} \\
\theta_{1}^{r} & \theta^{r} & \frac{\Gamma(r+n+1)}{\Gamma(r+2 n+2)}
\end{array}\right|=0 .
$$

Putting $q-p=\alpha, r-q=\beta$ in (19) $(a, \beta \geqslant 1)$

$$
\begin{aligned}
\Delta(\theta) \equiv & \theta^{\alpha+\beta}\left[\frac{\Gamma(q+n+1)}{\Gamma(q+2 n+2)}-\theta_{1}^{a} \frac{\Gamma(p+n+1)}{\Gamma(p+2 n+2)}\right]- \\
& -\theta^{a}\left[\frac{\Gamma(r+n+1)}{\Gamma(r+2 n+2)}-\frac{\Gamma(p+n+1)}{\Gamma(p+2 n+2)} \cdot \theta_{1}^{a+\beta}\right]+ \\
& +\left[\theta_{1}^{a} \frac{\Gamma(r+n+1)}{\Gamma(r+2 n+2)}-\theta_{1}^{a+\beta} \frac{\Gamma(q+n+1)}{\Gamma(q+2 n+2)}\right]=0 .
\end{aligned}
$$

We shall now show that this equations has a unique root $\theta_{0}$ lying in $\left(\theta_{1}, 1\right)$. We have

where

$$
\begin{aligned}
\Delta(0) & =\theta_{1}^{a}\left\{\frac{\Gamma(r+n+1)}{\Gamma(r+2 n+2)}-\theta_{1}^{\beta} \frac{\Gamma(q+n+1)}{\Gamma(q+2 n+2)}\right\} \\
& =\theta_{1}^{\alpha}\left\{\frac{\Gamma(q+n+1)}{\Gamma(r+2 n+2)}\right\}\left[(q+n+1)_{\beta}-\theta_{1}^{\beta}(q+2 n+2)_{\beta}\right] \\
& =\theta_{1}^{\alpha+\beta} \frac{\Gamma(q+n+1)}{\Gamma(r+2 n+2)} \cdot(q+n+1)_{\beta}\left\{\left(\frac{1}{\theta_{1}}\right)^{\beta}-\left(1+\frac{n+1}{q+n+1}\right)_{\beta}\right\}
\end{aligned}
$$

$$
\left(1+\frac{n+1}{q+n+1}\right)_{\beta}=\left(1+\frac{n+1}{q+n+1}\right)\left(1+\frac{n+1}{q+n+2}\right) \ldots\left(1+\frac{n+1}{q+n+\beta}\right) .
$$

Now

$$
\left(\frac{1}{\theta_{1}}\right)^{\beta}>\left(1+\frac{n+1}{q+n+1}\right), \quad \text { so } \quad \Delta(0)>0 \text {. }
$$


Also $\Delta\left(\theta_{1}\right)=0$ and

$$
\begin{aligned}
\Delta(1)=\theta_{1}^{a+\beta} & {\left[\frac{\Gamma(p+n+1)}{\Gamma(p+2 n+2)}-\frac{\Gamma(q+n+1)}{\Gamma(q+2 n+2)}\right]-} \\
- & \theta_{1}^{a}\left[\frac{\Gamma(p+n+1)}{\Gamma(p+2 n+2)}-\frac{\Gamma(r+n+1)}{\Gamma(r+2 n+2)}\right]+ \\
+ & {\left[\frac{\Gamma(q+n+1)}{\Gamma(q+2 n+2)}-\frac{\Gamma(r+n+1)}{\Gamma(r+2 n+2)}\right] }
\end{aligned}
$$

The right-hand side is a function of $\theta_{1}$ and corresponds to the left-hand side of (11), where we have shown that there exists a value $\dot{\theta}^{\prime}$ in $(0,1)$ such that $\Delta(1)>0$ for $\theta_{1}<\theta^{\prime}$. Also

$$
\begin{aligned}
& \Delta^{\prime}(\theta)=(\alpha+\beta) \theta^{\alpha+\beta-1} {\left[\frac{\Gamma(q+n+1)}{\Gamma(q+2 n+2)}-\theta_{1}^{a} \frac{\Gamma(p+n+1)}{\Gamma(p+2 n+2)}\right]-} \\
&-\alpha \theta^{a-1}\left[\frac{\Gamma(r+n+1)}{\Gamma(r+2 n+2)}-\theta_{1}^{a+\beta} \frac{\Gamma(p+n+1)}{\Gamma(p+2 n+2)}\right],
\end{aligned}
$$

so that

$$
\begin{aligned}
\Delta^{\prime}(1)=\theta_{1}^{a+\beta}\left[(\alpha+\beta)\left(\frac{1}{\theta_{1}}\right)^{\beta}\right. & \left\{\frac{\Gamma(q+n+1)}{\Gamma(q+2 n+2)} \cdot\left(\frac{1}{\theta_{1}}\right)^{a}-\frac{\Gamma(p+n+1)}{\Gamma(p+2 n+2)}\right\}- \\
& \left.-\alpha\left\{\left(\frac{1}{\theta_{1}}\right)^{\alpha+\beta} \cdot \frac{\Gamma(r+n+1)}{\Gamma(r+2 n+2)}-\frac{\Gamma(p+n+1)}{\Gamma(p+2 n+2)}\right\}\right]
\end{aligned}
$$

and

$$
\begin{aligned}
& \Delta^{\prime}\left(\theta_{1}\right)=(\alpha+\beta) \theta_{1}^{2 a+\beta-1} {\left[\left(\frac{1}{\theta_{1}}\right)^{a} \frac{\Gamma(q+n+1)}{\Gamma(q+2 n+2)}-\frac{\Gamma(p+n+1)}{\Gamma(p+2 n+2)}\right]-} \\
&-a \theta_{1}^{2 a+\beta-1}\left[\left(\frac{1}{\theta_{1}}\right)^{a+\beta} \frac{\Gamma(r+n+1)}{\Gamma(r+2 n+2)}-\frac{\Gamma(p+n+1)}{\Gamma(p+2 n+2)}\right] .
\end{aligned}
$$

We shall now show that

$$
\Delta^{\prime}(\mathbf{1})>0 \text { and } \Delta^{\prime}\left(\theta_{1}\right)<0 \text {. }
$$

The proof depends on the following inequality:

$$
\begin{aligned}
& \frac{a+\beta}{\alpha} \cdot A^{\beta}>\frac{\frac{\Gamma(r+n+1)}{\Gamma(r+2 n+2)} A^{a+\beta}-\frac{\Gamma(p+n+1)}{\Gamma(p+2 n+2)}}{\frac{\Gamma(q+n+1)}{\Gamma(q+2 n+2)} A^{\alpha}-\frac{\Gamma(p+n+1)}{\Gamma(p+2 n+2)}}>\frac{a+\beta}{a}, \\
& \text { for } \quad A \geqslant 3, \quad a \geqslant 1
\end{aligned}
$$

or

$$
\begin{aligned}
\frac{A^{\beta}}{\alpha}\{(p+n & \left.+1)_{a} A^{a}-(p+2 n+2)_{\alpha}\right\} \\
& >\frac{1}{\alpha+\beta} \cdot \frac{\Gamma(q+2 n+2)}{\Gamma(r+2 n+2)}\left\{(p+n+1)_{a+\beta} A^{a+\beta}-(p+2 n+2)_{a+\beta}\right\} \\
& >\frac{1}{\alpha}\left\{(p+n+1)_{a} A^{a}-(p+2 n+2)_{a}\right\}
\end{aligned}
$$


or

$$
\begin{aligned}
\frac{A^{\beta}\left\{A^{a}-\left(1+\frac{n+1}{p+n+1}\right)\right\}}{a\left(1+\frac{n+1}{p+n+1}\right)_{a}}>\frac{A^{a+\beta}-\left(1+\frac{n+1}{p+n+1}\right)_{a+\beta}}{(\alpha+\beta)\left(1+\frac{n+1}{p+n+1}\right)_{a+\beta}} & \frac{A^{a}-\left(1+\frac{n+1}{p+n+1}\right)_{a}}{a\left(1+\frac{n+1}{p+n+1}\right)_{a}}
\end{aligned}
$$

In other words

$$
A^{\beta} K(\alpha)>K(\alpha+\beta)>K(\alpha)
$$

where

$$
K(a)=\frac{A^{a}-\left(1+\frac{n+1}{p+n}+1\right)_{a}}{a\left(1+\frac{n+1}{p+n+1}\right)_{a}} .
$$

Since $\beta$ is a positive integer, it sufficient to prove the result for $\beta=1$. The right-hand side of the inequality is certainly true if

$$
\frac{A^{a+1}-\left(1+\frac{n+1}{p+n+1}\right)_{a+1}}{(a+1)\left(1+\frac{n+1}{p+n+1}\right)_{a+1}}>\frac{A^{a}-\left(1+\frac{n+1}{p+n+1}\right)_{a}}{a\left(1+\frac{n+1}{p+n+1}\right)_{a}}
$$

or

$$
\frac{A^{a}}{\left(1+\frac{n+1}{p+n+1}\right)_{a}}\left[\frac{A}{(a+1)\left(1+\frac{n+1}{p+n+a+1)}\right)}-\frac{1}{a}\right]>\frac{1}{a+1}-\frac{1}{a} .
$$

Since

$$
\frac{A}{1+\frac{n+1}{p+n+a+1}} \cdot \frac{1}{a+1}-\frac{1}{a}>\frac{1}{\alpha+1}-\frac{1}{\alpha}
$$

the inequality (26) is proved.

In order to prove the left-hand side of inequality $\left(25^{\prime}\right)$, we must show that:

$$
A^{a+1}\left[1+\frac{(n+1)(a+1)}{p+n+\alpha+1}\right]>\left(1+\frac{n+1}{p+n+1}\right)_{a+1}[(\alpha+1) A-\alpha] .
$$

We first observe that if (27) holds for $A=m$, then it also holds for $A>m$. Take

$$
\Phi(A)=A^{a+1}\left[1+\frac{(n+1)(a+1)}{p+n+a+1}\right]-\left(1+\frac{n+1}{p+n+1}\right)_{a+1}[(\alpha+1) A-\alpha]
$$


then

$$
\begin{aligned}
& \Phi^{\prime}(A)=(a+1)\left[\left\{1+\frac{(n+1)(a+1)}{p+n+a+1}\right\} A^{a}-\right. \\
& \left.-\left(1+\frac{n+1}{p+n+a+1}\right)\left(1+\frac{n+1}{p+n+1}\right)\right]>0,
\end{aligned}
$$

showing that $\Phi(A)$ is an increasing function of $A$, and this proves our assertion. (27) holds true if we show that

$$
A^{a+1}-(\overline{a+1} A-a)\left(1+\frac{n+1}{p+n+1}\right)_{a}>-\frac{a(n+1)}{p+2 n+a+2} \cdot A^{a+1} .
$$

The right-hand side being negative, it is sufficient to prove that

$$
A^{a+1}-((a+1) A-a)\left(1+\frac{n+1}{p+n+1}\right)_{a}>0
$$

which is certaintly true, since

$$
A^{a+1}>((\alpha+1) A-a) 2^{a}, \quad \text { for } \quad(A \geqslant 3, a \geqslant 3) .
$$

The cases $a=1$ and $a=2$ are verified as follows: For $a=1$, we should have

$$
\begin{aligned}
& {\left[A-\left(1+\frac{n+1}{p+n+1}\right)\right]^{2} } \\
&>\frac{n+1}{p+n+3}\left[\left(1+\frac{n+1}{p+n+1}\right)\left(1+\frac{n+1}{p+n+2}\right)-A^{2}\right] .
\end{aligned}
$$

Since the right-hand side is always negative for $A \geqslant 2$, while the left-hand side is positive, the inequality holds. For $a=2$, we should have

$$
A^{3}-(3 A-2)\left(1+\frac{n+1}{p+n+1}\right)\left(1+\frac{n+1}{p+n+2}\right)>-A^{3} \cdot \frac{2(n+1)}{p+2 n+4} .
$$

Proving the above for $A=2$ implies the proof for $A \geqslant 2$, so that we should have

$$
(n+1)\left[\frac{2 p+3 n+4}{(p+n+1)(p+n+2)}-\frac{4}{p+2 n+4}\right]<1,
$$

which reduces to proving

$$
p^{3}+(6 n+9) p^{2}+\left(6 n^{2}+18 n+14\right)>0,
$$

which is always true. Hence the inequality for $a=2$.

In these two cases we see that inequality (27) holds under less restrictive conditions $A \geqslant 2$. It appears that the inequality can be proved to be true for all $A \geqslant 2$ and $a \geqslant 1$. I have verified a few particular cases but the general proof seems to evade me. 
We have thus proved the inequality (25). On examining the expressions for $\Delta_{1}^{\prime}(1)$ and $\Delta_{1}^{\prime}\left(\theta_{1}\right)$ we at once get $(24)$. Also

$$
\begin{aligned}
& \dot{\Delta}_{1}^{\prime}(\theta)=\theta^{a-1} \theta_{1}^{a+\beta}\left[( a + \beta ) ( r + n + 1 ) _ { n + 1 } ( \frac { \theta } { \theta _ { 1 } } ) ^ { a + \beta } \left\{(p+n+1)_{n+1}\left(\frac{1}{\theta_{1}}\right)^{a}-\right.\right. \\
& \left.\left.-(q+n+1)_{n+1}\right\}-\alpha(q+n+1)_{n+1}\left\{(p+n+1)_{n+1}\left(\frac{1}{\theta_{1}}\right)^{\alpha+\beta}-(r+n+1)_{n+1}\right\}\right]
\end{aligned}
$$

which vanishes at $\bar{\theta}$ given by

$$
\vec{\theta}=\theta_{1}\left[\frac{a(q+n+1)_{n+1}\left\{(p+n+1)_{n+1}\left(1 / \theta_{1}\right)^{a+\beta}-(r+n+1)_{n+1}\right\}}{(a+\beta)(r+n+1)_{n+1}\left\{(p+n+1)_{n+1}\left(1 / \theta_{1}\right)^{a}-(q+n+1)_{n+1}\right\}}\right]
$$

and by (25) $\theta_{1}<\bar{\theta}<1$.

Hence from (21), (22), (24) we see that there exists a unique value $\theta_{0}>\bar{\theta}$ for which $\Delta_{1}(\theta)$ vanishes and the order of $R(h)$ is less than that of $h^{n+8+1}$ for this choice of $\theta$.

6. In connection with Art. 3, we have tired without success to show that the order of smallness of $R(h)$ is exactly $n+s+1$. In the case of $n=0$, this result had been proved by S. Gołąb and C. Olech [3]. For a general $n>0$, it would be necessary to show that if the order of $R(h)$ is $n+8+2$ then we are lead to a contradiction. For then we would have also

$$
\bar{\lambda}_{1} \theta^{s}+\lambda_{2}=1 /(s+n+1)_{n+1}
$$

which combined with (7) and (8) gives

$$
\left|\begin{array}{lll}
\theta^{p} & 1 & 1 /(p+n+1)_{n+1} \\
\theta^{q} & 1 & 1 /(q+n+1)_{n+1} \\
\theta^{s} & 1 & 1 /(s+n+1)_{n+1}
\end{array}\right|=0
$$

and on further simplification we may write it as

$$
\begin{aligned}
\left\{\frac{1}{(p+n+1)_{n+1}}-\frac{1}{(q+n+1)_{n+1}}-\theta^{a+\gamma}-\right. \\
-\left\{\frac{1}{(p+n+1)_{n+1}}-\frac{1}{(s+n+1)_{n+1}}\right\} \theta^{a}+ \\
+\left\{\frac{1}{(q+n+1)_{n+1}}-\frac{1}{(s+n+1)_{n+1}}\right\}=0
\end{aligned}
$$

where $\gamma=s-q$. We must now show that (30) and (11) cannot have a common root $\theta$.

In short, it reduces to showing that the equation

$$
K \theta^{x}=\lambda+\frac{\mu}{(x+q+n+1)_{n+1}}
$$


where

and

$$
\begin{aligned}
K & =\left[(q+n+1)_{n+1}-(p+n+1)_{n+1}\right] \theta^{a}>0, \\
\lambda & =\left[(q+n+1)_{n+1} \theta^{a}-(p+n+1)_{n+1}\right]<0
\end{aligned}
$$

$$
\mu=(p+n+1)_{n+1}(q+n+1)_{n+1}\left(1-\theta^{a}\right)>0
$$

has only three zeros $-a, 0$ and $\beta$.

A similar problem remains unsolved in connection with the problem treated in Art. 5 where we try to show that the order of smallness of $R(h)$ is exactly $n+s+1$. The case $n=0$ has been proved by $A$. Sharma [4]. As before, for a general $n>0$, we are led to a contradiction when we try to show that the order of $R(h)$ is $n+s+2$. Following the same technique, the problem is to prove that the equation

$$
\begin{aligned}
(s+n+1)_{n+1} \theta^{a+\gamma} \theta_{1}^{-\gamma}\left\{(p+n+1)_{n+1} \theta_{1}^{-a}-(q+n+1)_{n+1}\right\}- \\
-(q+n+1)_{n+1} \theta^{a}\left\{(p+n+1)_{n+1} \theta_{1}^{-a-\gamma}-(s+n+1)_{n+1}\right\}+ \\
+(p+n+1)_{n+1}\left\{(q+n+1)_{n+1} \theta_{1}^{-\gamma}-(s+n+1)_{n+1}\right\}=0
\end{aligned}
$$

and (20) cannot have a common root $\theta_{0}$ such that $\theta_{1}<\theta_{0}<1$.

I am grateful to Dr. A. Sharma for his valuable guidence in the preparation of this paper.

\section{References}

[1] N. Cioranescu, Une généralisation de la premiere formule de la moyenne et les polynômes de Tchebychef, C. R. Paris 206 (1938), p. 1782-1784.

[2] S. Goląb, Contribution à la formule Simpsonienne de quadralure approchée, Ann. Polon. Math. 1 (1954), p. 166-175.

[3] - et C. Olech, Contribution à la théorie de la formule Simpsonienne des quadratures approchées, Ann. Polon. Math. 1 (1954), p. 176.183.

[4] A. Sharma, On Golqb's contribution to Simpson's formula, Ann. Polon. Math. 3 (1957), p. 240-246.

DEPARTMENT OF MATHEMATICS \& ASTRONOMY, UNIVERSITY LUCKNOW, INDIA

Reģu par la Rédaction le 1. 2.1958 